\title{
Semmelweis and his thesis
}

Irvine Loudon

J R Soc Med 2005;98:555

Ignaz Phillip Semmelweis (1818-1865) is the most famous name in the history of obstetrics. He has been portrayed not only as a medical hero but also a martyr driven to madness and early death by the implacable opposition of his contemporaries. It is widely believed that he was the first person to discover that puerperal fever was contagious, that his discovery led to the virtual disappearance of the disease, and that his thesis The Etiology, the Concept and the Prophlyaxis of Childbed Fever (1860-1861) is one of the greatest publications on obstetrics ever written.

None of this is true. ${ }^{1}$ Others (notably Alexander Gordon of Aberdeen) had shown that puerperal fever was contagious some 40 years before Semmelweis. The mortality rate in lying-in hospitals did not fall but rose after Semmelweis' treatise was published, and his 'muchcited but seldom-read' thesis is immensely long (over 500 pages) and so badly written that it is almost unreadable.

Although the thesis contains passages of clearly written clinical detail, they are frequently interrupted by passages directed at his critics that are muddled, dogmatic and extremely quarrelsome. At one point he devoted 64 pages to a furious attack on Scanzoni, an obstetrician at Prague who had mildly questioned some of Semmelweis' beliefs. Semmelweis wrote his thesis 'feverishly, all in a great hurry... constantly writing fresh chapters, repeating portions without co-ordination, and constantly hurrying the manuscript off to the printers without revision'? Semmelweis wrote a second book in 1861 which consists entirely of intemperate attacks on his critics. ${ }^{3}$ It is clearly not a book by a sane and balanced medical scientist.

Medical Historian

E-mail: irvine.loudon@wuhmo.ox.ac.uk
Semmelweis deserves to be remembered for his observation in 1847 that making medical students wash their hands in a disinfectant solution before going from the post-mortem room to the lying-in wards of the Vienna lying-in hospital, led to a fall in mortality due to puerperal fever from a very high level to a moderate level. It was Semmelweis' misfortune to be so hypersensitive to criticism that, although his friends implored him to publish this work, he wrote nothing until he began his thesis in the late 1850 s. By this time it was clear that he was in the early stages of a psychosis. The nature of the psychosis is still uncertain (as is the cause of his death in a lunatic asylum) but it was probably a manic-depressive psychosis, or possibly syphilis - an occupational hazard of gynaecologists before surgical gloves were introduced.

After his death in 1865, Semmelweis was totally forgotten, not only in Vienna but also in Hungary where he was born and to which he returned in 1850. It was not until the late nineteenth century that the introduction of Listerian antisepsis applied to obstetrics led to a steep fall in deaths from puerperal fever. Only then was Semmelweis' work recalled and his faults forgotten. Sir William Sinclair's hagiographic biography, published in 1909, was only the first of a long series of publications that have portrayed Semmelweis as a faultless hero and his thesis as a masterpiece.

\section{REFERENCES}

1 Loudon Irvine. The Tragedy of Childbed Fever. Oxford: Oxford University Press, 2000:88-110

2 Sinclair Sir William. Semmelweis, His Life and His Doctrine. Manchester: Manchester University Press, 1909

3 Semmelwis IP. Open Letters to Sundry Professors of Obstetrics. 1861 